\title{
Enhancement of noisy signals by stochastic resonance
}

\author{
Zonghua Liu ${ }^{\text {a }}$, Ying-Cheng Lai ${ }^{\mathrm{a}, \mathrm{b}, *}$, Arje Nachman $^{\mathrm{c}}$ \\ a Department of Mathematics and SSERC, Arizona State University, Tempe, AZ 85287, USA \\ ${ }^{\mathrm{b}}$ Departments of Electrical Engineering and Physics, Arizona State University, Tempe, AZ 85287, USA \\ ${ }^{c}$ Air Force Office of Scientific Research, 801 North Randolph Road, Arlington, VA 22203, USA \\ Received 19 November 2001; received in revised form 20 February 2002; accepted 20 March 2002 \\ Communicated by A.R. Bishop
}

\begin{abstract}
We propose a scheme to detect signals immersed in strong, externally imposed, undesirable noise (jamming) by making use of the principle of stochastic resonance. The idea is to construct an array of simple nonlinear oscillators and to apply independent, modulating noise to each oscillator. We argue and provide evidence that the phenomenon of stochastic resonance, which is due to the interplay between nonlinearity and different noise sources, can enhance the detectability of the original signal. $\odot 2002$ Published by Elsevier Science B.V.
\end{abstract}

PACS: 05.40.-a; 05.45.Xt

Stochastic resonance is a nonlinear phenomenon that has attracted much recent attention [1-6]. Broadly speaking, stochastic resonance means that performances of the system, such as the response to periodic signals, can be enhanced by the noise and be made optimal at certain nonzero noise levels. This phenomenon is rather counter-intuitive, but the mechanism lies in the complex interplay between nonlinearity and stochasticity. In this Letter, we propose an approach, based on the principle of stochastic resonance, to an important problem in signal processing: detecting signals immersed in externally imposed, undesirable noise. This is in fact the problem of countering jamming. In particular, we propose a class of

\footnotetext{
* Corresponding author.

E-mail address: yclai@chaos1.la.asu.edu (Y.-C. Lai).
}

signal-processing devices by utilizing a nonlinear dynamical system that has the potential to exhibit robust stochastic resonance. Such a system can be simple and be built at low cost. The input signal consists of both the desired signal and jamming, for which a signal-tonoise ratio (SNR) can possibly be defined. A modulating noise signal is deliberately fed into the system. The system is so designed that, at some optimal levels of modulating noise, an output signal is yielded with a higher value of the SNR than that of the input signal.

In a typical situation where a single nonlinear element is utilized to generate stochastic resonance, high values of SNR are achieved only in a small range about the optimal noise level $D_{m}$. There are then two cases in the presence of jamming noise of amplitude $D_{J}: D_{J}<D_{m}$ and $D_{J}>D_{m}$. In the former case, the SNR can possibly be improved when one deliberately applies additive modulating noise of amplitude $D$ to 
the system such that the combined noise amplitude is about $D_{m}$. However, in the latter case where jamming is strong, applying additional noise cannot help improve the SNR. The difficulty here is due to the practical constraint that the adjustable parameter, namely the modulating-noise amplitude, can only strengthen the combined noise. In order to overcome this difficulty, it is necessary to introduce additional parameters to shape the stochastic resonance. Previous works indicate that systems consisting of an array of coupled (and uncoupled) nonlinear oscillators [3] can exhibit the phenomenon of extended stochastic resonance [4] in the sense that higher values of the SNR can be achieved in a wide range of the noise amplitude. We will use a similar strategy to address the problem of countering jamming.

In this Letter, we investigate stochastic resonance in the following class of systems of $N$ coupled nonlinear oscillators:

$$
\begin{aligned}
\frac{d \mathbf{x}_{i}}{d t}= & \mathbf{f}_{i}\left(\mathbf{x}_{i}\right)+K\left(\mathbf{X}-\mathbf{x}_{i}\right)+\mathbf{S}(t) \\
& +D_{J} \epsilon(t)+D \xi_{i}(t),
\end{aligned}
$$

where $i=1,2, \ldots, N, \mathbf{x}_{i} \in \mathcal{R}^{d}$ is a $d$-dimensional vector of the $i$ th oscillator with the nonlinear velocity field $\mathbf{f}_{i}\left(\mathbf{x}_{i}\right), \mathbf{X}=(1 / N) \sum_{i=1}^{N} \mathbf{x}_{i}$ is the ensemble average of all vectors (or the "mean field"), $K$ is the coupling strength, $\mathbf{S}(t)$ is the signal to be detected, $D_{J} \epsilon(t)$ is the noise term representing the jamming, and $D \xi_{i}(t)$ is the independent, Gaussian-white, modulating noise of amplitude $D$ applied to the $i$ th oscillator. The input signal is $\mathbf{x}^{\mathrm{in}}(t)=\mathbf{S}(t)+D_{J} \epsilon(t)$ and the output signal is $\mathbf{x}^{\text {out }}=\mathbf{X}(t)$ that typically consists of a signal $\mathbf{S}^{\text {out }}(t)$ related to the original signal $\mathbf{S}(t)$ and a noisy background signal $\mathbf{N}^{\text {out }}(t)$. With a proper arrangement of the system according to Eq. (1), it is hoped that, at some optimal levels of the modulating noise, the SNR of the output signal can be enhanced compared with that associated with the input signal. Intuitively, because of the statistical independence of the modulating noisy signals applied to different oscillators, an averaging process can help reduce the overall noisy background including the contribution from the jamming, thereby enhancing the SNR. Our principal result is that in a variety of arrangements of the system, the SNR can indeed be enhanced by tuning the modulating noise amplitude $D$. A practical device based on Eq. (1), such as a one constructed by using nonlinear electronic circuits, will then have the potential to reduce the influence of undesirable jamming.

It should be emphasized that the coupling scheme in the model system Eq. (1) is of the global or mean-field type. Our result that the detectability of periodic signals can be enhanced by modulating noise is thus valid under this setting. In fact, as our analysis indicates, the mean-field type of coupling is important for reducing the noisy background of the signal so as to enhance the SNR. In this regard, other coupling schemes, such as the nearest-neighbor type, are expected not to be as effective.

There have been previous works on signal detection using stochastic resonance [7-11]. The general situation considered in these works is that there is only one noise source. As such, the SNRs of the input and output signals are defined with respect to this common noise source. It is shown that, if the system is approximately linear so that its processing of the signal can be described by the traditional linear response theory, then the gain in the SNR cannot be greater than one [9]. A fairly recent work on a single bistable system indicates that for this system, even in the nonlinear regime, the SNR gain is less than one. We note, however, that in somewhat more general settings, the SNR gain can exceed one in the nonlinear regime [7]. The problem that we address here is quite different from these previous ones in that we have two independent noise terms: one corresponding to the jamming and another to the deliberately applied control noise. The meaning of the SNR for the input signal in our case, which is the ratio of the signal power to the power of the jamming, is thus very different from that in the existing work [7-11]. As we will show in this Letter, our coupled configuration of nonlinear oscillators, with two independent noise sources, can in general yield SNR gains that exceed unity.

We first present numerical results with the following system of $N$ coupled, two-dimensional FitzHughNagumo oscillators:

$$
\begin{aligned}
\frac{d x_{i}}{d t}= & \frac{1}{\delta}\left[x_{i}\left(x_{i}-a\right)\left(1-x_{i}\right)-y_{i}\right] \\
& +K\left(X-x_{i}\right)+g\left(x_{i}\right)\left[D \xi_{i}(t)\right], \\
\frac{d y_{i}}{d t}= & x_{i}-y_{i}-b+\left[S(t)+D_{J} \epsilon(t)\right],
\end{aligned}
$$


where $i=1, \ldots, N, g\left(x_{i}\right)$ is a function, $\delta, a$ and $b$ are parameters in the FitzHugh-Nagumo oscillator. If $g\left(x_{i}\right)=1$, the modulating noise $D \xi_{i}(t)$ is additive, otherwise if $g\left(x_{i}\right)$ is not a constant, the modulating noise is regarded as being multiplicative. The individual FitzHugh-Nagumo oscillator is an excitable dynamical system which has been the paradigm for studying stochastic resonance [2-5]. We consider the following periodic signal: $S(t)=r \sin \omega_{0} t$ with amplitude $r$ and frequency $\omega_{0}$. Because of the two independent noise terms, we find it convenient to use the Milshtein method [12] to numerically integrate the set of stochastic differential equations in (2). For concreteness, we choose $r=0.34$ (arbitrarily). Other parameters are: $\delta=0.005, a=0.5, b=0.15, K=1.0$ and $\omega_{0}=15.0$. The jamming is assumed to be a Gaussianwhite noise with amplitude varying from 0.1 to 0.3 . The total integrated power of the input signal is $\pi r^{2}$, so the SNR of the input signal is $\pi r^{2} / D_{J}^{2}$. The frequency of the input signal is $f_{0}=\omega_{0} / 2 \pi \approx 2.38$, so the power spectrum of the output signal $X(t)$ contains a peak at the same frequency. The signal can be detected by using a bandpass filter centered at $f_{0}$. The SNR of the output is determined by the height of the peak relative to the noisy background at $f_{0}$. Fig. 1(a) shows, for $D_{J}=0.1$ and three different system sizes $N$, the SNR versus the amplitude of the modulating
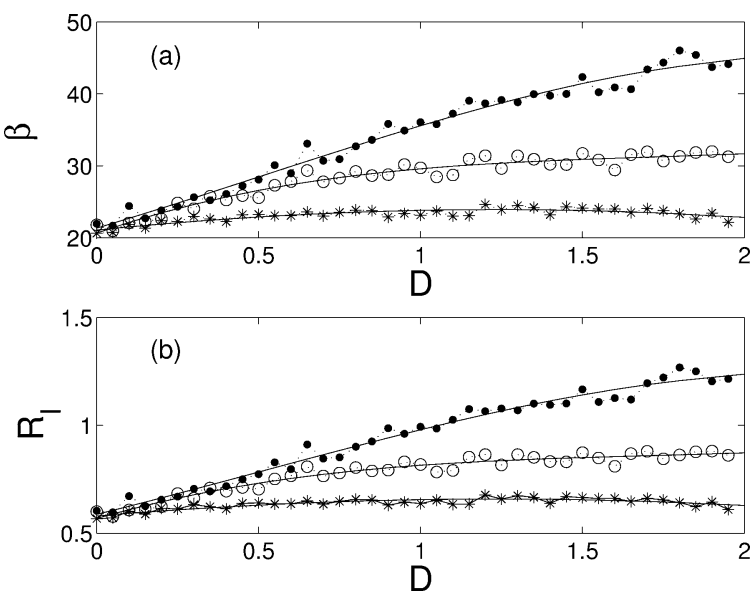

Fig. 1. For additive, Gaussian-white modulating noise in Eq. (2) with a sinusoidal input signal: (a) the SNR versus $D$, the amplitude of the modulating noise; (b) $R_{I}$, improvement of the SNR measure, versus $D$. Filled circles, open circles, and stars correspond to $N=50, N=10$ and $N=2$, respectively. noise, where the closed circles, open circles, and stars denote system sizes of $N=50,10$ and 2, respectively. For large system size, we observe extended stochastic resonance in the sense that the SNR keeps increasing as the amplitude of the modulating noise is increased, which is desirable.

An important measure is how much the SNR can be improved. For $D_{J}=0.1$, the SNR of the input signal is about 36 . We notice that in the range of the modulating noise considered, the maximum SNR that can be achieved is about 50 , so indeed, there is a somewhat nontrivial gain in the SNR. Fig. 1(b) shows the SNR improvement $R_{I}$ versus $D$, which is defined to be the ratio between the SNRs of the output and input signals. Apparently, for large $N(N=50)$, the value of $R_{I}$ can be above unity for $D \gtrsim 1.0$. This indicates that an array of nonlinear oscillators can be utilized to suppress the influence of jamming.

What is the optimal system size $N$ ? Fig. 2 shows, for $D_{J}=0.1$ and $D=1.5, R_{I}$ versus $N$. Apparently, the value of $R_{I}$ first increases with $N$ and then saturates at about $N=50$, indicating that no substantial improvement in the SNR can be achieved for $N \gtrsim 50$. Thus, for the particular parameter setting in Figs. 1 and $2, N \approx 50$ seems to be the optimal system size. This implies that using very large arrays of coupled oscillators may not be advantageous from the standpoint of enhancing the SNR of the output. In general, we observe a similar behavior for other settings of the system including multiplicative modulating noise.

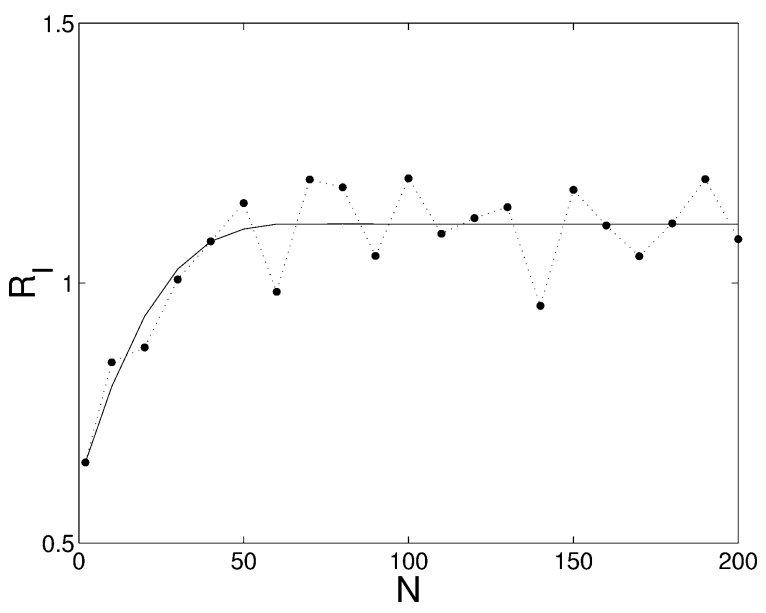

Fig. 2. Improvement $R_{I}$ of SNR versus the system size $N$ for $D_{J}=0.1$ and $D=1.0$. 
The results above suggest that for the system of coupled FitzHugh-Nagumo oscillators, Eq. (2), the SNR of the output signal appears to increase with the amplitude $D$ of the modulating noise. Does this mean that larger noise is always advantageous? The answer is perhaps "no" because any physical system has only a finite noise tolerance. The point here is that the results in Figs. 1 and 2 represent only the amplitude property of the system in response to a noisy sinusoidal signal. Other important quantities characterizing the overall response of the system are the following correlation measures suggested by Collins et al. [5]: $C_{0}=\overline{S(t) R(t)}, C_{1}=C_{0} /\left[\overline{S^{2}(t)}\right]^{1 / 2}[\overline{R(t)-\overline{R(t)}}]^{1 / 2}$, where $S(t)$ is a zero-mean input signal, $R(t)$ is the response function determined by, say, the bursting or the firing rate of the excitable system, and $\overline{R(t)}$ denotes the time average of $R(t)$. Both $C_{0}$ and $C_{1}$ are scalar measures of the coherence between the input and output signals. For the special case of a periodic driving, $C_{0}$ is the transferred signal strength, and $C_{1}$ is related to the SNR [5]. Computing the correlation measures, however, requires the knowledge of the response function $R(t)$, the determination of which can be nontrivial [5]. We notice, however, that the main characteristic of a bursting time series is the time intervals between the successive bursts. Thus, for an output bursting signal $X(t)$, we set a threshold, say $X_{\text {th }}$, and define the following quantized response function: $\Theta(t)=+1$ if $X(t)>X_{\text {th }}$ (bursting phase) and $\Theta(t)=-1$ if $X(t)<X_{\text {th }}$ (silent phase). Due to noise, details of $X(t)$ in the bursting and silent phases can be quite complicated, but our definition of the response function $\Theta(t)$ ignores these details. The following correlation measure can then be introduced:

$C=\frac{\overline{\Theta(t) S(t)}}{\sqrt{\overline{|S(t)|^{2}\left[\Theta^{2}(t)-\langle\Theta\rangle^{2}\right]}}}$.

Notice that the above measure $C$ can also be used to characterize stochastic resonance with respect to aperiodic signals.

Fig. 3(a),(b) show, for fixed jamming strength and for fixed system size, respectively, the correlation measure $C$ versus $D$, where the input signal is the sinusoidal one, and $X_{\text {th }}=0.25$. Specifically, for Fig. 3(a), the jamming strength is fixed at $D_{J}=0.1$ and different curves correspond to $N=50$ (filled circles), $N=10$ (open circles) and $N=2$ (stars). For Fig. 3(b), the
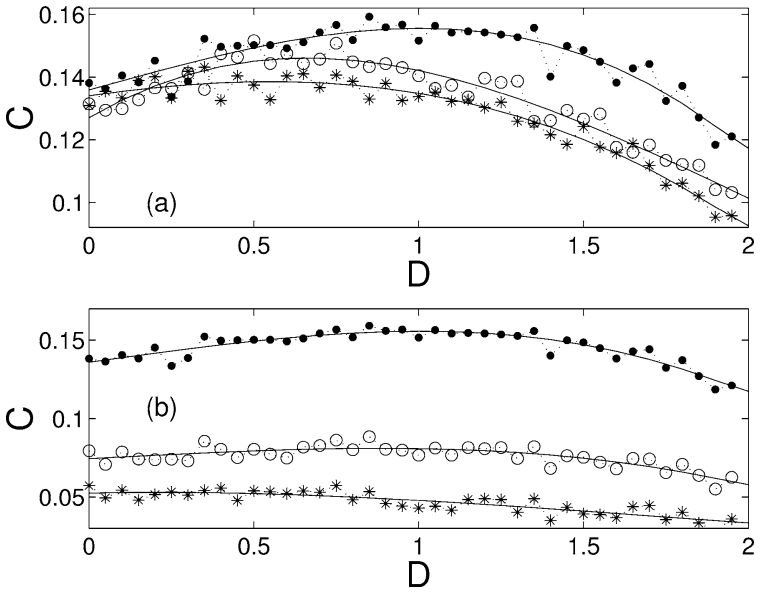

Fig. 3. Correlation measure $C$ versus $D$ for (a) fixed jamming strength and (b) fixed system size. See text for details.

system size is fixed at $N=50$ and the curves correspond to $D_{J}=0.1$ (filled circles), $D_{J}=0.2$ (open circles) and $D_{J}=0.3$ (stars). There is apparently a resonance-like behavior in $C$ in the sense that the correlation measure first increases with $D$, then decreases with $D$, and reaches a maximum for $D \approx 1.0$. Larger noise results in smaller value of $C$, which is undesirable. Numerically, we find a similar behavior with jammed aperiodic signals.

We now give a heuristic theory for stochastic resonance in the coupled system Eq. (2). To gain insight, we notice the following basic ingredients for stochastic resonance:

(1) a threshold mechanism in the response of the system, such as the bursting mechanism in a typical excitable system or that due to a potential barrier in a mechanical system;

(2) a subthreshold periodic signal, one which is not sufficiently strong to trigger a burst or in the case of potential barrier, to push the particle to cross the barrier;

(3) the existence of noise.

Particle motion in a one-dimensional double potential well, subject to a weak signal and noise, is perhaps the simplest system satisfying the above three conditions. We are thus led to considering the following one-dimensional model describing each individual 
nonlinear oscillator:

$\frac{d x}{d t}=-\frac{\partial U(x)}{\partial x}+S_{0} \cos \left(\omega_{0} t\right)+D_{J} \epsilon(t)+D \xi(t)$,

where the potential function is of the following quadrupolar form so that it possesses a double-well structure:

$U(x)=-\frac{a x^{2}}{2}+\frac{b x^{4}}{4}$

( $a$ and $b$ are constants), $S_{0} \cos \left(\omega_{0} t\right)$ is the signal to be detected, $D_{J} \epsilon(t)$ is the jamming, and $D \xi(t)$ is the modulating noise term. The corresponding system of coupled double-potential-well oscillators can then be written, as follows:

$$
\begin{aligned}
\frac{d x_{i}(t)}{d t}= & a x_{i}-b x_{i}^{3}+S_{0} \cos \left(\omega_{0} t\right)+D_{J} \epsilon(t) \\
& +D \xi_{i}(t)+K\left[X(t)-x_{i}(t)\right]
\end{aligned}
$$

where $i=1, \ldots, N$ and the mean field

$X(t)=\sum x_{i}(t) / N$,

is the output signal of the full coupled system.

A detailed theory for stochastic resonance in the one-dimensional model of a single oscillator, via the solutions to the associated Fokker-Planck equation, is given by McNamara and Wiesenfeld [6]. Our point is that, in an approximate sense, the system of $N$ coupled double-well potential systems can be casted into a simple one-dimensional form, as follows. Summing over all variables in the coupled system yields the following equation for the output of the system $X(t)$ :

$$
\frac{d X}{d t}=a X-b^{\prime} X^{3}+S^{\prime}(t)+D_{J} \epsilon(t)+\frac{1}{N} \sum_{i} \xi_{i}(T),
$$

where $b^{\prime} \equiv b N^{2}, S^{\prime}(t)=S_{0} \cos (\omega t)+Q(t)$, and $Q(t) \equiv N^{2} X^{3}-\sum x_{i}^{3} / N$. With these changes in the coefficient $b$ and in the signal $S(t)$, Eq. (7) is equivalent in form to the one-dimensional model Eq. (4). The equivalent potential is $U_{0}^{\prime}=a^{2} / 4 b^{\prime}=U_{0} / N^{2}$, meaning that the particle can go over the barrier more easily so as to enhance the SNR. More specifically, the signal and noise powers can be written down explicitly, from which the SNR can be computed. For instance, Fig. 4 shows the relationship between the SNR and $N$ for a typical set of parameter values in the potential model, which is consistent with the numerical curve in Fig. 2.

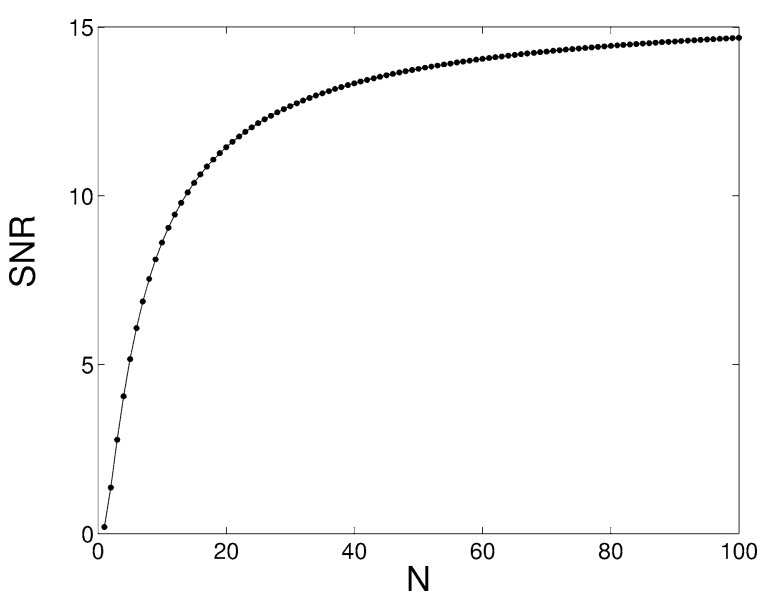

Fig. 4. Theoretical SNR versus the system size $N$ for a typical set of parameters in the potential model.

In summary, motivated by the problem of combating jamming, we have investigated stochastic resonance in excitable dynamical systems subject to multiple, independent noisy disturbances. We suggest a scheme employing an array of coupled FitzHughNagumo oscillators for detecting signals immersed in vast jamming noise whose spectrum covers entirely that of the signal (in-band noise). Our results indicate that under fairly general settings, a marked improvement in the SNR of the output signal can be achieved. Theoretically, our results are novel in the sense that stochastic resonance in coupled excitable systems, in the presence of multiple noise sources, is a new perspective in this area. On the applied side, our study can potentially lead to an alternative methodology for countering jamming.

We remark that fairly recently, information-theoretic measures have been introduced to characterize the responses of stochastic-resonant systems to aperiodic signals [13-15]. For instance, Stokes [15] considers a summing array of threshold devices and studies how $I$, the average mutual information, a concept from the traditional information theory [16], is influenced by noise. The general finding is that there is a resonant behavior in $I$ versus the noise amplitude in the sense that $I$ achieves its maximum at an optimal noise level. In these studies [13-15], a common feature in the settings is again that the input signal is not contaminated by noise or, there is no jamming. For our problem with both jamming and modulating noise, such an information-theoretic measure can be still used. 


\section{Acknowledgement}

This work was supported by AFOSR under Grant No. F49620-98-1-0400.

\section{References}

[1] R. Benzi, A. Sutera, A. Vulpiani, J. Phys. A 14 (1981) L453; R. Benzi, G. Parisi, A. Sutera, A. Vulpiani, J. Appl. Math. 43 (1983) 565 .

[2] Stochastic resonance is such an extensive field that even a partial list of the literature is prohibitive here. For review of stochastic resonance, see, for example:

P. Jung, Phys. Rep. 234 (1993) 175;

F. Moss, D. Pierson, D. O'Gorman, Int. J. Bifur. Chaos 4 (1994) 1383;

K. Wiesenfeld, F. Moss, Nature (London) 373 (1995) 33;

L. Gammaitoni, P. Hänggi, P. Jung, F. Marchesoni, Rev. Mod. Phys. 70 (1998) 223.

[3] P. Jung, U. Behn, E. Pantazelou, F. Moss, Phys. Rev. A 46 (1992) R1709.

[4] J.J. Collins, C.C. Chow, T.T. Imhoff, Nature (London) 376 (1995) 236

P.C. Gailey, A. Neiman, J.J. Collins, F. Moss, Phys. Rev. Lett. 79 (1997) 4701.

[5] J.J. Collins, C.C. Chow, T.T. Imhoff, Phys. Rev. E 52 (1995) R3321;

C. Heneghan, C.C. Chow, J.J. Collins, T.T. Imhoff, S.B. Lowen, M.C. Teich, Phys. Rev. E 54 (1996) R2228;
D. Nozaki, J.J. Collins, Y. Yamamoto, Phys. Rev. E 60 (1999) 4637.

[6] B. McNamara, K. Wiesenfeld, Phys. Rev. A 39 (1989) 4854.

[7] M.E. Inchiosa, A.R. Bulsara, Phys. Rev. E 52 (1995) 327; F. Chapeau-Blondeau, X. Godivier, Phys. Rev. E 55 (1997) 1478;

M.E. Inchiosa, A.R. Bulsara, A.D. Hibbs, B.R. Whitecotton, Phys. Rev. Lett. 80 (2000) 1381.

[8] M.E. Inchiosa, A.R. Bulsara, Phys. Rev. E 53 (1996) R2021.

[9] A. Neiman, L. Schimansky-Geier, F. Moss, Phys. Rev. E 56 (1997) R9.

[10] D.F. Russell, L.A. Wilkens, F. Moss, Nature 402 (1999) 291.

[11] P. Hänggi, M.E. Inchiosa, D. Fogliatti, A.R. Bulsara, Phys. Rev. E 62 (2000) 6155.

[12] P.E. Kloeden, E. Platen, Numerical Solution of Stochastic Differential Equations, Springer, Berlin, 1992.

[13] M.E. Inchiosa, J.W.C. Robinson, A.R. Buslara, Phys. Rev. Lett. 85 (2000) 3369;

J.W.C. Robinson, J. Rung, A.R. Buslara, M.E. Inchiosa, Phys. Rev. E 63 (2001) 011107.

[14] I. Goychuk, P. Hänggi, Phys. Rev. E 61 (2000) 4272; I. Goychuk, Phys. Rev. E 64 (2001) 021909.

[15] N.G. Stocks, Phys. Rev. Lett. 84 (2000) 2310; N.G. Stocks, Phys. Rev. E 63 (2001) 041114; N.G. Stocks, Phys. Lett. A 279 (2001) 308; N.G. Stocks, R. Mannella, Phys. Rev. E 64 (2001) 030902.

[16] See, for example: A. Feinstein, Foundations of Information Theory, McGrawHill, New York, 1958;

M.F. Reza, An Introduction to Information Theory, Dover, New York, 1994 